\title{
Metástasis poco habituales del carcinoma diferenciado de tiroides
}

\author{
J. C. FERRER GARCÍA, J. F. MERINO TORRES, J. L. PONCE MARCO*, \\ F. PIÑ́N SELLÉS
}

Servicio de Endocrinología y *Cirugía General. Hospital Universitario La Fe. Valencia

\author{
LESS COMMON SITES OF METASTASES IN DIFFERENTIATED \\ THYROID CANCER
}

\section{RESUMEN}

El carcinoma diferenciado de tiroides metastatiza fundamentalmente en pulmón y hueso, y con menos frecuencia en cerebro, hígado y piel. Se describen las características clínicas, terapéuticas e histológicas del tumor primario en tres casos de metástasis de carcinoma diferenciado de tiroides en lugares poco habituales. En dos de los casos se trataba de un carcinoma folicular, el primero presentó una metástasis en glándula suprarrenal derecha y el segundo en riñón izquierdo. En los dos casos, inicialmente, sólo se realizó una hemitiroidectomía sin tratamiento posterior con $\mathrm{I}^{131}$, y en uno de ellos no se trataron cifras elevadas de tiroglobulina en el seguimiento por ser los rastreos con $I^{131}$ negativos. En el tercer caso el diagnóstico anatomopatológico inicial fue de carcinoma papilar, procediéndose a tiroidectomía total más ablación de restos con $\mathrm{I}^{131}$ y tratamiento supresor con levotiroxina, sin embargo, la evolución posterior fue desfavorable presentando metástasis en lugares típicos y atípicos como fue en coroides. La revisión de la pieza histológica del último caso reveló una variedad de células altas del carcinoma papilar de tiroides. En conclusión, ante un carcinoma diferenciado de tiroides cuyo tratamiento inicial no haya sido el correcto, es posible encontrar metástasis en los lugares habituales y también en otros más infrecuentes. Determinadas variedades del carcinoma papilar de tiroides como es la de células altas, presentan un comportamiento especialmente agresivo y pueden metastatizar en lugares poco habituales.

PALABRAS CLAVE: Metástasis infrecuentes del carcinoma de tiroides. Carcinoma papilar de células altas de tiroides. Carcinoma papilar y metástasis en coroides. Carcinoma folicular y metástasis renal y suprarrenal.

\begin{abstract}
Distant metastases of differentiated thyroid cancer are usually loca lised in the lung and bone; less common sites of metastases are the brain, liver, and skin. To find it in other sites it is exceptional. This work shows the clinical, histologycal and therapeutical characteristic of primary tumour in three cases of non-typical distant metastases of differentiated thyroid cancer. Follicular thyroid carcinoma was displayed in two cases, the first presented one metastases in right adrenal gland and the second in left kidney. Both cases were initially treated only with hemithyroidec tomy, without posterior radioiodine. In one case, elevated serum thyro globulin did not receive radioiodine treatment because Whole-Body Scintigraphy was negative. The thirst case was a papillary thyroid carci noma treated with total thyroidectomy, ablative radioiodine and sup pressor therapy with levothyroxine. However, posterior evolution was unfavourable and tumour presented metastases in common and less com mon sites like choroid. Histologycal study showed tall cell variant of papillary thyroid carcinoma. In summary, when the initial treatment of well-differentiated thyroid carcinoma it is inaccurate, it is possible to find common and less common sites metastases. Some variants of papi llary thyroid carcinoma, like tall cell, have a worse prognosis and they can present metastases in less common sites.
\end{abstract}

KEY WORDS: Non-typical metastases of thyroid carcinoma. Tall cell papillary thyroid carcinoma. Papillary carcinoma and metastases in choroid. Follicular carcinoma and metastases in kidney and adrenal gland.

Ferrer García JC, Merino Torres JF, Ponce Marco JL, Piñón Sellés F. Metástasis poco habituales del carcinoma diferenciado de tiroides. An Med Interna (Madrid) 2002; 19: 579-582.

\section{INTRODUCCIÓN}

El carcinoma diferenciado de tiroides (CDT), tanto en su variedad papilar como folicular, es una de las neoplasias con mejor pronóstico, con una supervivencia a los cinco años que oscila entre el 90-95\%. Actualmente constituye menos del $1 \%$ de todos los procesos cancerígenos, con una incidencia anual que varía entre 0,5-10 casos por 100.000 habitantes $(1,2)$. Se suele presentar como un nódulo tiroideo aunque no es infrecuente la presencia, adicional o como único hallazgo, de adenopatías cervicales, supraclaviculares y/o mediastínicas. No obstante, estas adenopatías, a diferencia de las que aparecen en otras neoplasias, no indican la existencia de metástasis a distancia $(3,4)$.

Las metástasis a distancia del CDT no son habituales. Aparecen con una frecuencia del 10 al $15 \%$ del total $(3,4)$ y

Trabajo aceptado: 13 de septiembre de 2001

Correspondencia: J. Fco. Merino Torres. Servicio de Endocrinología. Hospital Universitario La Fe. Avda. de Campanar, 21. 46009 Valencia. e-mail: merino_jfr@gva.es 
se han relacionado con edad avanzada, sexo masculino y extensión local del tumor (5). Cuando metastatiza lo hace fundamentalmente en pulmón y hueso. Las metástasis más habituales son las pulmonares (65-70\%), sobre todo en pacientes jóvenes y en la variedad papilar (3). Las óseas aparecen en sujetos de edad más avanzada, son más frecuentes en la variedad folicular y tienen peor pronóstico $(1-4,6)$. Otras localizaciones de metástasis más infrecuentes (2-7\%) son cerebro, hígado y piel $(3,4,7)$. El hallazgo de metástasis en otros lugares suele ser un hecho excepcional, generalmente relacionado con tratamientos incorrectos, retrasos diagnósticos y/o variedades histológicas agresivas del tumor.

Se presentan tres casos en los que han objetivado metástasis de CDT en localizaciones poco habituales, como son al nivel de la glándula suprarrenal, riñón y coroides.

\section{CASOS APORTADOS}

Se han revisado los CDT diagnosticados en nuestro hospital desde enero de 1988 a marzo de 1999, identificando tres casos con metástasis en lugares poco habituales, al nivel de glándula suprarrenal, riñón y coroides ocular. Se analizan las características clínicas, estadio de DeGroot al diagnóstico (8), variedad histológica y tratamiento aplicado (Tabla I).

Caso 1: Varón de 68 años diagnosticado de nódulo tiroideo autónomo hiperfuncionante tratado con $\mathrm{I}^{131}$ en cuatro ocasiones hasta completar una dosis total de $133 \mathrm{mCi}$. Cuatro años después del diagnóstico inicial y ante la imposibilidad de controlar la hiperfunción se practicó una hemitiroidectomía cuya anatomía patológica fue de carcinoma folicular, en un estadio III de DeGroot. Ocho meses más tarde se administró una dosis ablativa de $100 \mathrm{mCi}$ de $\mathrm{I}^{131}$ y se inició tratamiento con 1tiroxina a una dosis de $1,75 \mu \mathrm{g} / \mathrm{kg} /$ día, con cifras de TSH nunca inhibidas. Dos años después se objetivó un ascenso progresivo de la tiroglobulina, alcanzando un valor máximo de $329 \mathrm{ng} / \mathrm{ml}$ sin que se objetivaran metástasis en la TAC torácica ni en el rastreo óseo. A los tres años del diagnóstico histológico, ante la persistencia de cifras elevadas de tiroglobulina, se administró $150 \mathrm{mCi}$ de $\mathrm{I}^{131}$ y en el rastreo corporal total posterior se confirmó un área de captación abdominal derecha, por lo que fue remitido al servicio de endocrinología. La TAC abdominal identificó una masa suprarrenal derecha (Fig. 1 A y B). El estudio histológico tras adrenalectomía confirmó la metástasis de carcinoma folicular de tiroides.
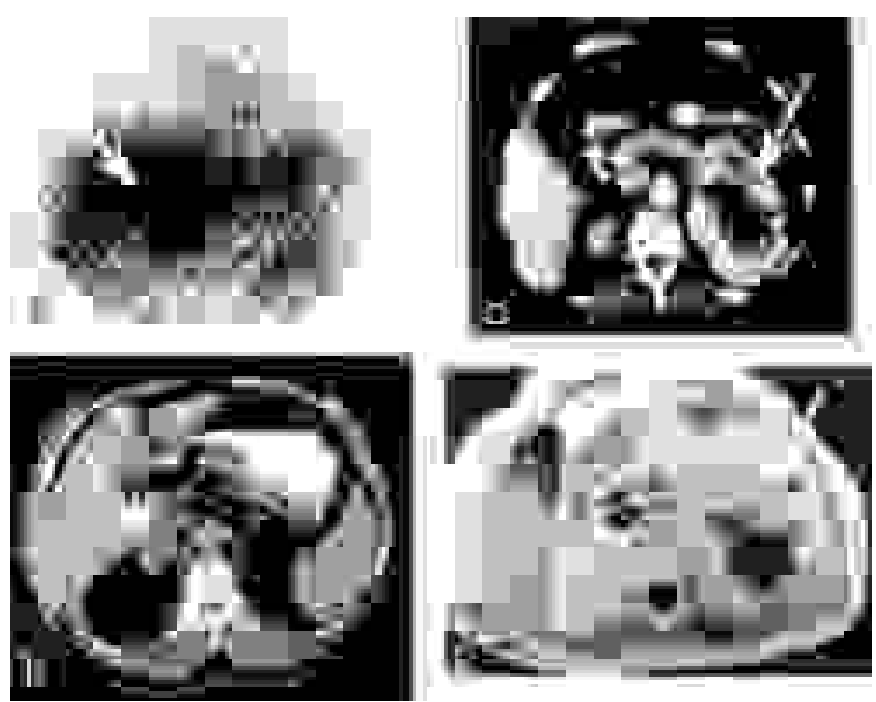

Fig. 1. M etástasis al nivel de la glándula suprarrenal derecha $(A$ y $B$ ), se observa la captación de $~^{131}$ en el rastreo corporal total (1 A, flecha) y la imagen nodular en la TAC abdominal. Metástasis a nivel renal izquierdo vista con TAC (1 C) y RM (1 D) abdominal.

Caso 2: Mujer de 58 años diagnosticada inicialmente de bocio multinodular normo-funcionante y tratada con hemitiroidectomía. El informe anatomopatológico fue de bocio adenomatoso coloide con adenoma folicular tipo trabecular. A los dos años, ante una recidiva local, se practicó tiroidectomía subtotal siendo el informe anatomopatológico de carcinoma folicular invasor. Se inició tratamiento supresor con 1-tiroxina a dosis de $2,4 \mu \mathrm{g} / \mathrm{kg} /$ día y no se administró tratamiento con $\mathrm{I}^{131}$. Cinco años más tarde, tras consultar por lumbalgia, se practicó RM en la que se evidenció una masa renal izquierda (Fig. 1 C y D) y se remitió al servicio de endocrinología. El estudio citológico confirmó la existencia de metástasis de carcinoma folicular de tiroides, por lo que se completó la cirugía de la glándula tiroides y de la metástasis renal, que confirmó el diagnóstico citológico y el estadio IV de DeGroot. Con posterioridad, ante la existencia de metástasis óseas y pulmonares, se administró tratamiento con $\mathrm{I}^{131}$ hasta haber completado $515 \mathrm{mCi}$ y se mantuvo el tratamiento supresor con 1-tiroxina. La evolución fue favorable y mantiene cifras de tiroglobulina de $3 \mathrm{ng} / \mathrm{ml}$.

TABLA I

CARACTERÍSTICAS CLÍNICAS, TERAPÉUTICAS E HISTO LÓ GICAS DE LOS CASO S CLÍNICOS

\begin{tabular}{|c|c|c|c|}
\hline & Caso 1 & Caso 2 & Caso 3 \\
\hline $\begin{array}{l}\text { Enfermo } \\
\text { Tratamiento inicial }\end{array}$ & Varón 68 años & M ujer 58 años & Varón 36 años \\
\hline $\begin{array}{l}\text { Cirugía } \\
\text { Vaciamiento }\end{array}$ & Hemitiroidectomía & Hemitiroidectomía & Tiroidectomía total \\
\hline $\begin{array}{l}\text { ganglionar } \\
\text { Tratamiento con }\end{array}$ & No & No & Sí \\
\hline $\begin{array}{l}\text { I131 } \\
\text { Tiroidectomía total }\end{array}$ & $\begin{array}{c}\text { No } \\
\text { A los } 4 \text { años del }\end{array}$ & $\begin{array}{c}\text { No } \\
\text { A los } 7 \text { años del }\end{array}$ & $\begin{array}{c}\text { Sí } \\
\text { Desde el diagnóstico }\end{array}$ \\
\hline$+1^{131}+$ Levotiroxina & diagnóstico & diagnóstico & \\
\hline $\begin{array}{l}\text { Rastreos } \varnothing \text { con Tiroglobulina alta } \\
\text { Anatomía Patológica }\end{array}$ & $\begin{array}{c}\text { No } \\
\text { Carcinoma Folicular }\end{array}$ & $\begin{array}{c}\text { No } \\
\text { Carcinoma Folicular }\end{array}$ & $\begin{array}{c}\text { Sí } \\
\text { Carcinoma papilar } \\
\text { variedad de células altas }\end{array}$ \\
\hline M etástasis atípicas & $\begin{array}{l}\text { Glándula suprarrenal } \\
\text { derecha (figura } 1 \mathrm{~A}, \mathrm{~B} \text { ) }\end{array}$ & $\begin{array}{l}\text { Riñón izquierdo } \\
\text { (figura 1 C, D) }\end{array}$ & Coroides (figura $2 \mathrm{~A}, \mathrm{~B}$ ) \\
\hline
\end{tabular}



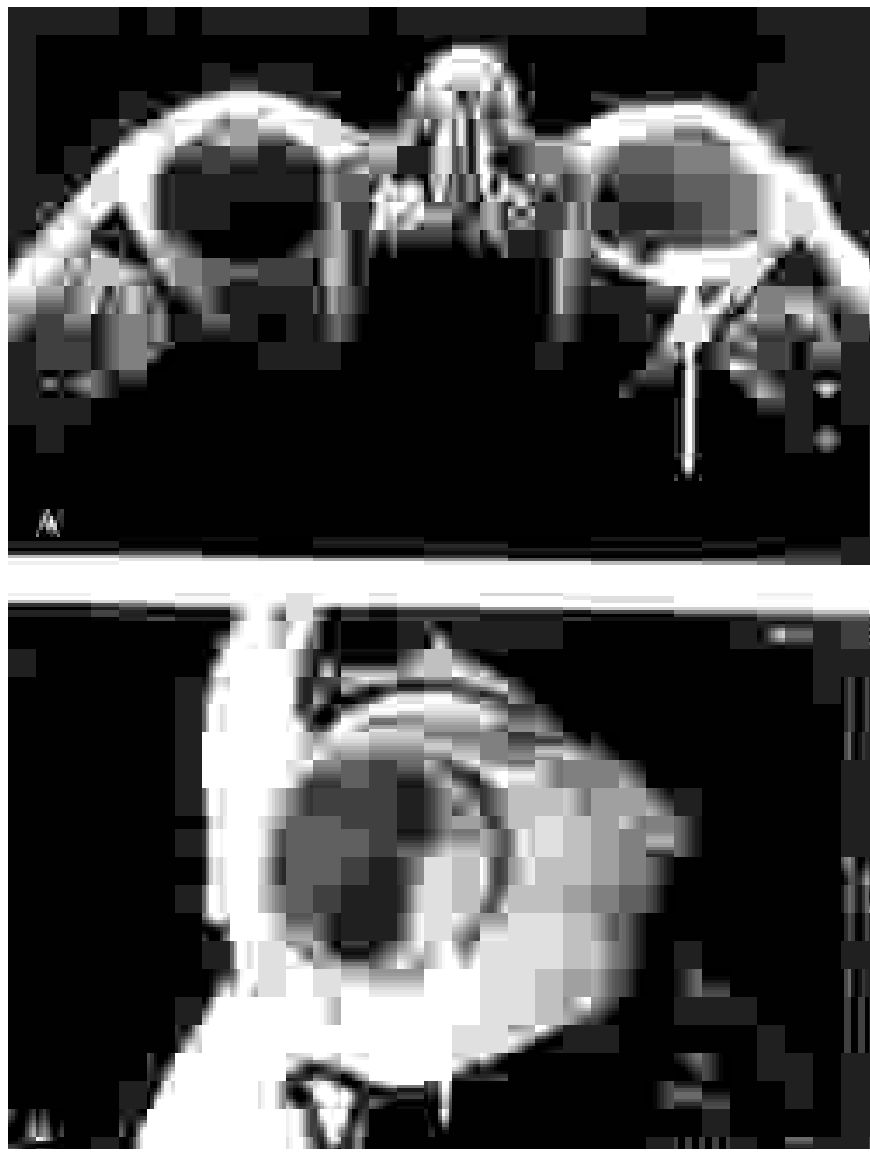

Fig. 2. M etástasis localizada en coroides en una imagen obtenida con RM orbitaria (flecha).

Caso 3: Varón de 36 años remitido por nódulo gammagráficamente frío en lóbulo tiroideo izquierdo. El estudio citológico fue compatible con carcinoma papilar de tiroides, por lo que se procedió a tiroidectomía total y vaciamiento ganglionar bilateral, confirmándose el diagnóstico citológico, con un estadio III de DeGroot. Con posterioridad se administró $100 \mathrm{mCi}$ de $\mathrm{I}^{131}$ y se inició tratamiento supresor con 1-tiroxina a dosis de $2,6 \mu \mathrm{g} / \mathrm{kg} /$ día. A los cuatro años del diagnóstico se evidenció un aumento de la tiroglobulina $(36,5 \mathrm{ng} / \mathrm{ml})$ con rastreo corporal total con $\mathrm{I}^{131}$ negativo. Se administró dosis ablativa de $\mathrm{I}^{131}$, pero la evolución posterior fue desfavorable presentando metástasis al nivel de pulmón, hueso, cerebro y coroides ocular (Fig. 2 A y B). Ante la mala evolución, se revisó la pieza histológica, de acuerdo con los nuevos criterios, y se informó como variedad de células altas del carcinoma papilar de tiroides. Se procedió a tratamiento quirúrgico de las metástasis accesibles, entre ellas vitrectomía posterior izquierda, cuya histología demostró metástasis de carcinoma papilar tiroideo de células altas. El tratamiento se completó con radioterapia local en las zonas indicadas, dosis ablativa de $\mathrm{I}^{131} \mathrm{y}$ quimioterapia con vincristina, 4 epirrubicina y cisplatino.

\section{DISCUSIÓN}

El tratamiento del CDT ha sido objeto de numerosas publicaciones (3,8-10). Se basa en tres pilares fundamentales y sucesivos: tiroidectomía total con revisión de las cadenas ganglionares, radioyodo y tratamiento supresor con l-tiroxina. La no correcta secuencia ensombrece el pronóstico del CDT y favorece la aparición de metástasis en localizaciones típicas y atípicas (casos 1 y 2). No obstante, en los raros casos de varie- dades histológicas especialmente agresivas, aún aplicando correctamente el tratamiento, pueden evolucionar desfavorablemente (caso 3).

El tratamiento quirúrgico sigue siendo controvertido. La práctica más utilizada es la tiroidectomía total en todos aquellos casos de tumores de tamaño superior a $1 \mathrm{~cm}(10,11)$. Existe un gran número de estudios que demuestran la relación entre la extensión de la cirugía y el pronóstico de la enfermedad (12). Hay y cols. demostraron un incremento en la recurrencia a los 20 años de seguimiento según se realizase tiroidectomía total o unilateral (5\% versus $20 \%$ respectivamente) (13). Tanto en el caso 1 como en el 2, inicialmente se realizó tiroidectomía subtotal. La cirugía debe incluir revisión de las cadenas ganglionares y extirpación de aquellas macroscópicamente afectas $(2,3,14,15)$. En el caso de la variedad papilar, dado que hasta $2 / 3$ de los casos tienen ganglios afectos al diagnóstico y que en más del $80 \%$ son del compartimento central, aún sin afectación macroscópica, se vaciará dicho compartimento y se revisará el resto $(2,3,14,15)$. En la variedad folicular el $35 \%$ tienen ganglios afectos al diagnóstico, por lo que se aconseja vaciamiento ganglionar si son palpables en el acto quirúrgico o el diagnóstico es cierto antes o durante la intervención $(2,3,14,15)$.

Entre los 7-14 días tras la cirugía se realizará un rastreo corporal total con $\mathrm{I}^{131}$. La dosis para el rastreo diagnóstico es variable, siendo la pauta más frecuentemente empleada de 3 a $5 \mathrm{mCi}$. Esta técnica tiene una sensibilidad del 60-80\% y una alta especificidad que oscila entre el 98 y el $100 \%$ (16). Entre cuatro y siete semanas tras la cirugía y después de haber confirmado una TSH por encima de $30 \mathrm{mUI} / \mathrm{L}$, se administrará una dosis ablativa de $50-150 \mathrm{mCi}$ de $\mathrm{I}^{131}$ para eliminar posibles restos cervicales $(17,18)$. Finalmente, el tratamiento con l-tiroxina tiene como objetivo mantener una TSH suprimida dado el carácter trófico de esta sobre las células tiroideas. Ello supone una dosis aproximada de 2,2 a 2,8 $\mu \mathrm{g} / \mathrm{kg} /$ día (17). En el primer caso no se mantuvieron dosis supresoras de l-tiroxina.

El seguimiento del CDT debe incluir además del control clínico la determinación de tiroglobulina, anticuerpos antitiroglobulina y TSH $(3,19-21)$. La tiroglobulina es una glicoproteína producida únicamente por las células foliculares tiroideas, ya sean normales o neoplásicas. Su aumento en el seguimiento de un enfermo diagnosticado y tratado de carcinoma diferenciado de tiroides indica la presencia de tejido tiroideo, aun cuando el rastreo corporal total con $\mathrm{I}^{131}$ a dosis diagnósticas sea negativo $(3,22)$. El hallazgo de rastreos negativos coincidiendo con cifras reiteradamente elevadas de tiroglobulina y una vez descartado falso positivo por presencia de anticuerpos antitiroglobulina, obliga a administrar una dosis terapéutica de $\mathrm{I}^{131}$, realizando con posterioridad nuevo rastreo corporal total que con frecuencia sí que objetivará metástasis dada la mayor actividad de radioyodo administrada $(3,22,23)$. No obstante, existen autores que están abogando últimamente por la realización de gammagrafía con 99mTc-MIBI como alternativa en estos casos, pues se han observado captaciones positivas que no lo eran con el rastreo habitual con radioyodo (24). El tratamiento con radioterapia estaría indicado en casos de extensión metastásica, aunque no existen datos que demuestren mejoría en la supervivencia (18). La quimioterapia, basada en antraciclinas y cisplatino, tiene escasa utilidad y se reserva para tumores diseminados o aquellos que no responden a otros tratamientos como ocurrió en el caso 3 .

En los casos 1 y 2 el tratamiento fue incorrecto. La cirugía inicial fue incompleta y no incluyó la extirpación de las cade- 
nas ganglionares. La administración de radioyodo posterior se demoró al menos 6 meses respecto a la pauta más utilizada. Además, la dosis de 1-tiroxina empleada fue menor de lo aconsejable y mantenía cifras de TSH entre 0,5 y 1,0 mUI/L. En el caso 2 se practicó una cirugía en tres tiempos. Las dos primeras intervenciones fueron parciales y no incluyeron vaciamiento ganglionar. Tras estos dos primeros actos quirúrgicos no se administró tratamiento con radioyodo y fue tras la tercera intervención, cuando se extirpó la totalidad de restos tiroideos y se procedió a la resección de adenopatías, cuando se administró una dosis ablativa de $\mathrm{I}^{131}$; ya en estadio IV de DeGroot.

En el caso 3 el tratamiento administrado fue correcto de acuerdo con la mayoría de consensos. Sin embargo, la existencia de una variedad especialmente agresiva del carcinoma papilar de tiroides condicionó su peor evolución $(3,25-27)$. El carcinoma papilar de tiroides puede clasificarse en diferentes variedades histológicas entre las que se incluyen la encapsulada, folicular, de células altas, de células columnares, de células claras y esclerosante difuso. Entre las variantes más agresivas se encuentran la de células altas, células columnares y esclerosante difusa $(3,25-27)$.

En conclusión, la existencia de metástasis de carcinoma diferenciado de tiroides en localizaciones atípicas (que excluyen pulmón, hueso, sistema nervioso central, hígado y piel) es un hecho excepcional. Sin embargo, ante un CDT cuyo tratamiento inicial no haya sido el correcto, es posible encontrar metástasis en los lugares habituales y también en otros más infrecuentes. Del mismo modo, determinadas variedades del carcinoma papilar de tiroides como es la de células altas, presentan un comportamiento especialmente agresivo y pueden metastatizar en lugares poco habituales.

\section{Bibliografía}

1. Shaha AR, Shah JP, Loree TR. Differentiated thyroid cancer presenting initially with distant metastasis. Am J Surg 1997; 174: 474-6.

2. Parkin DM, Muir CS, Whelan SL, Gao YT, Ferlay J, Powell J. Cancer incidence in five continents. International Agency for Research on cancer. Lyon, France 1992; 120 (6).

3. Schlumberger MJ. Papillary and follicular thyroid carcinoma. N Eng J Med 1998; 338: 297-307.

4. Hoie J, Stenwig AE, Kullman G, Lindegaard M. Distant metastases in papillary thyroid cancer: a review of 91 patients. Cancer 1988; 61: 1-6.

5. Sato N, Oyamatsu M, Koyama Y, Emura I, Tamiysa I, Hatakeyama K. Do the level of nodal disease according to the TNM classification and the number of involved cervical nodes reflect prognosis in patients with differentiated carcinoma of the thyroid gland?. J Surg Oncol 1998; 69: 151-5.

6. Dinneen SF, Valimaki MJ, Bergstralh EJ, Goellner JR, Gorman CA, Hay ID. Distant metastases in papillary thyroid carcinoma: 100 cases observed at one institution during 5 decades. J Clin Endocrinol Metab 1995; 80: 2041-5.

7. Schlumberger M, Challeton C, De Vathaire F, et al. Radioctive iodine treatment and external radiotherapy for lung and bone metastases from thyroid carcinoma. J Nucl Med 1996; 37: 598-605.

8. De Groot LJ, Kaplan EL, McKormick M, Straus FH. Natural history, treatment and course of papillary thyroid carcinoma. J Clin Endocrinol Metab 1990; 71: 414-24.

9. Singer PA, Cooper DS, Daniels GH, et al. Treatment guidelines for patients with thyroid nodules and well-differentiated thyroid cancer. Arch Intern Med 1996; 156: 2165-72.

10. AACE clinical practice guidelines for the management of thyroid carcinoma. Endocr Pract 1997; 3: 60-71.

11. Solomon BL, Wartofsky L, Burman KD. Current trends in the management of well differentiated papillary thyroid carcinoma. J Clin Endocri nol Metab 1996; 81: 333-9.

12. Bellantone R, Lombardi CP, Boscherini M, et al. Prognostic factors in differentiated thyroid carcinoma: a multyvariate analysis of 234 consecutive patients. J Surg Oncol 1998; 68: 237-41.

13. Hay ID, Bergstralh EJ, Goellner JR, Ebersold JR, Grant CS. Predicting outcome in papillary thyroid carcinoma: development of a reliable prognostic scoring system in a cohort of 1779 patients surgically treated at one institution during 1940 through 1989. Surgery 1993; 114: 1050-8.

14. Mann B, Burh HJ. Lymph node dissection in patients with differentiated thyroid carcinoma--who benefits? Langenbecks Arch Surg 1998; 383: $355-8$.
15. Kelemen PR, Van Herle AJ, Giuliano AE. Sentinel lymphadenectomy in thyroid malignant neoplasms. Arch Surg 1998; 133: 288-92.

16. McDougall IR. Whole-Body Scintigraphy with Radioiodine-131. A comprehensive list of false positives with some examples. Clin Nucl Med 1995; 20: 869-75.

17. Sorge-Van Boxel RAJ, Van Eck-Smit BLF, Goslings BM. Comparison of serum thyroglobulin, $131 \mathrm{I}$ and $201 \mathrm{Tl}$ scintigraphy in the postoperative follow-up differentiated thyroid cancer. Nucl Med Comm 1993; 14: 365 .

18. Mazzaferri EL, Young RL. Papillary thyroid carcinoma: a 10 year follow up report of the impact of therapy in 576 patients. Am J Med 1981; 70: 511-18.

19. Chigot JP. Management of metastases. Medullary cancer excluded. Ann Endocrinol (Paris) 1997; 58: 335-7.

20. Bayer MF, McDougall IR, Kriss JP. Retrospective study of serum thyroglobulin measurements performed in patients with differentiated thyroid carcinoma at the Stanford University thyroid clinic between 1979 and 1985. In Hufner M, Reinewrs C (eds): Thyroglobulin and thyroglobulin antibodies in the follow-up of thyroid cancer and endemic goiter. Stugartt, Geoege Thieme Verlag 104-11.

21. Filesi M, Signori A, Ventroni G, Melacrinis FF, Ronga G. Role of initial iodine-131 whole body scan and serum thyroglobulin in differentiated thyroid carcinoma metastases. J Nucl Med 1998; 39: 1542-6.

22. Pineda JD, Lee T, Ain K, Reynolds JC, Robbins J. Iodine-131 therapy for thyroid cancer patients with elevated thyroglobulin and negative diagnostic scan: J Clin Endocrinol Metab 1995; 80: 1488-92.

23. Miyamoto S, Kasagi K, Misaki T, Alan MJ, Konishi J. Evaluation of technetium-99mMIBI scintigraphy in metastatic differentiated thyroid carcinoma. J Nucl Med 1997; 79: 564-73.

24. Tsang RW, Brierley JD, Simpson WJ, Panzarella T, Gosporadowicz MK, Sutcliffe SB. The effects of surgery, iodine and external radiation therapy on the clinical outcome of patients with differentiated thyroid carcinoma. Cancer 1998; 88: 375-88.

25. Johnson TL, Lloyd RV, Thompson NW, Beierwaltes WH, Sisson JC. Prognostic implications of the tall cell variant of papillary thyroid carcinoma. Am J Surg Pathol 1988; 12: 22.

26. Yunta PJ, Ponce JL, Prieto M, Merino JF, Sancho-Fornos S. The importance of a tumor capsule in columnar cell thyroid carcinoma: a report of two cases and review of the literature. Thyroid 1999; 9: 815-9.

27. Hawk WA, Hazard JB. The many appearances of papillary carcinoma of the thyroid. Clev Clin Q 1976; 43: 207. 